\title{
LESSON 141
}

\author{
Accents \\ Examples: \\ acute (') \\ grave (') \\ circumflex $\left({ }^{\wedge}\right)$ \\ diaeresis or umlaut ( ") \\ cedilla $($, \\ tilde $(\sim)$
}

Welsh

PAPER: A4.

French

PAPER: A4.

UNIT 36
If a typist finds that she has a lot of work to type in a foreign language and this means typing a large number of accents, she can obtain a typewriter fitted with the required accents. The accent keys are fitted instead of such keys as fractions which may not be required. Many modern machines used for typing foreign languages are fitted with a 'dead' key which does not act on the carriage spacing escapement. This means that if an accent is required the 'dead' key must be struck first, the accent typed and then the required letter must be typed. If there is no 'dead' key the backspacer must be used. If a machine does not have accent keys, use the comma for the cedilla (c) and put in the other accents later with a pen.

The Welsh Examinations Board offers a choice of a passage in English or Welsh for the copy test.

Try typing a foreign language as a concentration exercise.

Hwyrach bydd rhai ohonnoch sydd ddim yn Gymraeg yn meddwl mae go ryfedd yw gofyn i chwi deipio rhywbeth sydd $i$ chwi yn iaith dramor. Bydd hwn yn brawf da $i$ chwi, yn mynnu sylw llawnach nag arferol. Os yr ydych yn Gymraeg, mwy na thebyg fe fydd hwn mor hawdd $i$ chwi ag y mae yn Saesneg. Mae'r awdur wedi cynnwys hwr er mwyn gwella cywirdet y Saeson, ac i roi i'r ddau, y sais a'r Cymro, y cyfle i ymarfer cyfran sydd yn cynnwys acennau ynddo. Gellwch deipio nifero'r acennau, ond gyda'r lleill bydd rhaid defnyddio ysgrifbin. Rhyw amser, yn y dyfodol, tybed bydd ysgrifennyddion i gyd yn gorfod teipio cryn dipyn o waith mewn iaith wahanol i'w mhamiaith. Felly ni fydd $y$ math hwn o ymarferiad yn fwy dieithr nag arfer cywir.

Demandez à la plupart des gens ce qu'ils savent de la France et vous trouverez probablement que tout ce qu'ils savent, c'est que Paris est la capitale de la France et que la France produit des vins célèbres. Tout le monde a entendu parler de Napoléon Bonaparte et de la Révolution Francaise, mais bien peu davantage. Pour les Anglais la France est simplement le pays de l'autre côté de la Manche qui vaut bien une visite en excursion ou en vacances.

Tous les écoliers apprennent comment Guillaume le Conquérant a gagné en 1066 la bataille de Hastings et comment le pauvre Harold a été tué par une flèche qui lui a pénétré l'oeil. C'etait la bataille entre les Normands et les Anglais dont le résultat a été que le Duc de Normandie est devenu Roi d'Angleterre et que ses vassaux allaient ensuite coloniser et gouverner l'Angleterre. Voilà pourquoi il y a eu une grande infusion de sang francais dans la race anglaise à cette époque, et pourquoi on trouve en Angleterre bien des gens qui portent des noms d'origine francaise. 